\title{
Печеночная энцефалопатия:
}

\section{вопросы, актуальные для клинической практики}

М.В. Маевская, https://orcid.org/0000-0001-8913-140X, mvmaevskaya@me.com

Первый Московский государственный медицинский университет имени И.М. Сеченова (Сеченовский Университет); 119991, Россия, Москва, ул. Погодинская, д. 1, корп. 1

\section{Резюме}

Печеночная энцефалопатия (ПЭ) остается одним из наиболее серьезных осложнений цирроза печени. Разнообразие ее клинических проявлений создает определенные сложности в оптимальной диагностике прямо у постели больного и выборе терапии. В обзоре представлены новые знания и положения последних лет по вопросу клинической работы с пациентами, страдающими ПЭ на фоне цирроза печени. В клинической практике у пациентов с подозрением на явную, клинически выраженную ПЭ нормальная концентрация аммиака может быть использована для исключения данного диагноза. Напротив, высокая концентрация аммиака в отсутствие клинических признаков ПЭ не должна служить критерием данного диагноза и руководством для назначения лечения. Отдельный вопрос для обсуждения - скрытая ПЭ. Самый простой и доступный тест для скрининга скрытой ПЭ и оценки эффективности терапии, который может выполнить как врач, так и человек, ухаживающий за пациентом, - это тест на клички животных. Пациенты со скрытой ПЭ нуждаются в лечении, которое аналогично по своим подходам явной ПЭ. Диагностика явной ПЭ и методы ее терапии четко разработаны. Согласно российским рекомендациям, в зависимости от особенностей течения заболевания у конкретного пациента в качестве препаратов первой линии могут быть использованы лактулоза, рифаксимин, L-орнитин-L-аспартат, что применимо к лечению как явной, так и скрытой ПЭ. Основные дискутируемые вопросы по ведению ПЭ при циррозе печени касаются диагностической роли аммиака, оптимальной диагностики и стратегии терапии скрытой ПЭ, терапии выбора как для явной, так и скрытой ПЭ. По всем этим вопросам существуют мнения экспертов и согласительные документы. Лечение явной и скрытой ПЭ проводится по одинаковым принципам. Препараты выбора: лактулоза, рифаксимин и L-орнитин-L-аспартат.

Ключевые слова: печеночная энцефалопатия, нерешенные вопросы, диагностика, концентрация аммиака, тест на клички животных, лечение, препараты выбора

Для цитирования: Маевская М.В. Печеночная энцефалопатия: вопросы, актуальные для клинической практики. Медицинский совет. 2021;(15):104-108. https://doi.org/10.21518/2079-701X-2021-15-104-108.

Конфликт интересов: автор заявляет об отсутствии конфликта интересов.

\section{Hepatic encephalopathy: issues relevant to clinical practice}

Marina V. Maevskaya, https://orcid.org/0000-0001-8913-140X, mvmaevskaya@me.com

Sechenov First Moscow State Medical University (Sechenov University); 1, Bldg. 1, Pogodinskaya St., Moscow, 119991, Russia

\begin{abstract}
Hepatic encephalopathy $(\mathrm{HE})$ remains one of the most serious complications of liver cirrhosis. Its clinical spectrum sometimes creates difficulties in the optimal diagnosis at the patient's bedside and treatment. To present new data on the field of clinical management of cirrhotic patients with hepatic encephalopathy. The role of ammonia in the diagnosis of HE is still under discussion. In clinical practice, in patients with suspected overt HE, normal ammonia concentration can be used to exclude this diagnosis. In contrast, a high concentration of ammonia in the absence of clinical signs of HE should not serve as a criterion for this diagnosis and as a guide for treatment. A separate issue for discussion is the covert HE. The simplest and most affordable test for screening for covert HE and evaluating the effectiveness of therapy is the animal naming test, which can be done on bedside by physician or caregivers. Patients with covert HE need treatment that is similar in approach to overt HE. The diagnosis of overt $\mathrm{HE}$ and the methods of its therapy are well known. According to Russian recommendations, depending on the disease course in a certain patient, lactulose, rifaximin, L-ornithine L-aspartate can be used as first-line drugs, which is applicable to the treatment of both overt and latent PE. The main issues on the management of HE in liver cirrhosis relate to the diagnostic role of ammonia, optimal diagnosis and treatment strategy for covert HE, therapy of choice for both overt and latent HE. There are expert opinions and consensus documents on all these issues. Treatment of overt and latent PE is carried out according to the same principles. Drugs of choice: lactulose, rifaximin and L-Ornithine L-Aspartate.
\end{abstract}

Keywords: hepatic encephalopathy, unresolved issues, diagnosis, ammonia concentration, animal naming test, treatment, drugs of choice

For citation: Maevskaya M.V. Hepatic encephalopathy: issues relevant to clinical practice. Meditsinskiy sovet = Medical Council. 2021;(15):104-108. (In Russ.) https://doi.org/10.21518/2079-701X-2021-15-104-108.

Conflict of interest: the author declares no conflict of interest. 


\section{ВВЕДЕНИЕ}

Печеночная энцефалопатия (ПЭ) остается одним из наиболее серьезных осложнений цирроза печени. Разнообразие ее клинических проявлений создает определенные сложности в оптимально быстрой и правильной диагностике прямо у постели больного. В международном сообществе основным направляющим деятельность врачей документом в условиях повседневной практики служат клинические рекомендации. Относительно ПЭ они были созданы Американской и Европейской ассоциациями по изучению печени [1]. Положения этого документа учтены в российских рекомендациях по ведению пациентов с циррозом печени [2]. Однако с момента их написания прошло много лет, накоплены новые знания и опыт. Эти новые знания и положения обобщены в нескольких работах, ориентированных на оптимизацию именно клинической работы с пациентами, страдающими ПЭ: 1) консенсус международного общества по изучению ПЭ и метаболизма азота (ISHEN: The International Society for Hepatic Encephalopathy and Nitrogen Metabolism) от 2020 г. [3]; 2) обзорная статья мировых экспертов в вопросах ПЭ «Новый взгляд на классификацию, патофизиологию и терапию ПЭ» [4]; положения консенсуса экспертов в ведении пациентов с декомпенсированным циррозом печени, подготовленные Европейской ассоциацией по изучению печени и опубликованные в J. Hepatology в 2021 г. [5]. Они положены в основу новых дополненных рекомендаций по ведению пациентов с циррозом печени Российского общества по изучению печени, которые сейчас готовятся к публикации и принятию МЗ РФ.

Данный обзор представляет новые знания и положения последних лет по вопросу клинической работы с пациентами, страдающими ПЭ на фоне цирроза печени.

\section{ПАТОГЕНЕЗ ПЕЧЕНОЧНОЙ ЭНЦЕФАЛОПАТИИ: ОСНОВНЫЕ ПОЛОЖЕНИЯ}

Традиционно ПЭ подразделяется на явную (с неврологическими и/или психиатрическими отклонениями, которые могут быть обнаружены клинически) и скрытую (с отклонениями, которые выявляются с помощью нейропсихических или нейрофизиологических тестов).

Считается, что в основе патогенеза ПЭ лежат следующие основные факторы: 1) повышение содержания аммиака в крови (гипераммониемия) в связи с избыточным поступлением в кровь азотсодержащих веществ, синтезируемых кишечными бактериями и недостаточной обезвреживающей функцией гепатоцитов, 2) портосистемное шунтирование крови. Серьезную роль в патогенезе ПЭ играют тяжесть повреждения печени, степень выраженности воспаления и/или оксидативного стресса, возраст, ассоциированные заболевания (сахарный диабет, почечная недостаточность), тяжесть и характер провоцирующего ПЭ фактора.

Астроциты поглощают аммиак, участвующий в реакции превращения глутамата в глутамин; избыток последнего при гипераммониемии приводит к разбуханию астроцитов и тканевому отеку. Уменьшение содержания глутамата в астроцитах сопровождается нарушением его нейротрансмиссии с участием NMDA-рецепторов, а также повышением активности ГАМКергических процессов. Вероятнее всего, эти события лежат в основе когнитивного дефицита, судорожных пароксизмов и других клинических проявлений ПЭ [5, 6].

Несмотря на длительный период изучения ПЭ, по-прежнему остаются вопросы, требующие внесения ясности: корректная диагностика как клинически выраженной, так и скрытой ПЭ; роль определенных факторов ее патогенеза и, соответственно, диагностических инструментов, выбор оптимальных лечебных мер. Отдельные вопросы: тактика ведения пациентов с минимальной ПЭ и амбулаторный режим, а именно контроль за уровнем сознания, эффективностью лечения ПЭ в домашних условиях.

\section{АММИАК В ДИАГНОСТИКЕ ПЕЧЕНОЧНОЙ ЭНЦЕФАЛОПАТИИ}

В вопросах диагностики ПЭ продолжает обсуждаться роль аммиака. При циррозе печени повышенная концентрация аммиака в артериальной крови ассоциирована с тяжелыми формами ПЭ. Пациенты с его более высоким уровнем демонстрируют больше осложнений, включая глубокую ПЭ ( $p=0,055)$, судороги ( $p=0,006)$, отек мозга $(p=0,020)$, потребность в ИВЛ ( $p<0,001)$. Особое значение этот показатель имеет при острой печеночной недостаточностью: уровень аммиака в артериальной крови в момент госпитализации > 124 мкмоль/л служит прогностическим фактором летальности с чувствительностью 78,6\%, специфичностью 76,3\% и диагностической точностью 77,5\%. Уровень аммония при поступлении пациента с острой печеночной недостаточностью - независимый предиктор летальности (OR - 10,9) [7]. У пациентов с циррозом печени уровень аммиака 79,5 мкмоль/л служит прогностическим фактором 28-дневной летальности с чувствительностью и специфичностью 68,1\% и 67,4\% соответственно. Уровень аммиака $\geqslant 79,5$ мкмоль/л ассоциируется с большей частотой органной недостаточности, а именно: печеночной ( $p=0,004)$, системы коагуляции $(p<0,001)$, почечной ( $p=0,004)$, дыхательной $(p<0,001)$. Отсутствие положительной динамики в уровне аммония к 5-му дню госпитализации ассоциируется с высокой летальностью (70,6\%) [8].

Таким образом, уровень аммиака коррелирует не только с тяжестью ПЭ, но и с органной недостаточностью, а также служит прогностическим фактором летального исхода. Это делает данный параметр важным биомаркером и терапевтической мишенью.

Однако в повседневной практической деятельности диагностическое значение концентрации аммиака имеет определенные ограничения. Во-первых, большое значение играет, какая исследуется кровь: артериальная, венозная или капиллярная и, соответственно, вариации в нормальных значениях параметра. Сама техника забора крови требует навыка. Венозная кровь берется в пробирку со стабилизатором, охлаждается и должна быть исследована в лаборатории в течение 30-60 мин. Капиллярный 
аммиак лучше всего измерять в крови, взятой из мочки уха. Если кровь берется из кончика пальца, то даже небольшая примесь пота приводит к артефакту и значительному завышению показателя. У пациентов с циррозом печени и тяжелой ПЭ, требующей госпитализации В отделения интенсивной терапии, уровень аммиака более чем в 50\% случаев может быть в пределах нормы [9]. Более того, помимо ПЭ, при циррозе печени на уровень аммиака оказывают влияние такие события, как прием пищи с высоким содержанием белка [10] и эпизоды голодания, желудочно-кишечное кровотечение, трансъюгулярное внутрипеченочное портосистемное шунтирование (TIPS); концентрация аммиака повышается при снижении функции почек [3] и т. д.

Bсе эти данные позволяют сформулировать положения для практического применения такого метода, как исследование концентрации аммиака. У пациентов с подозрением на явную, клинически выраженную ПЭ нормальная концентрация аммиака может быть использована для исключения данного диагноза вследствие высокой $(0,81)$ отрицательной прогностической ценности данного показателя [4]. Обратная ситуация: высокая концентрация аммиака в отсутствие клинических признаков ПЭ не должна служить критерием данного диагноза и руководством для назначения лечения. Диагноз клинически выраженной ПЭ основан на объективном исследовании пациента и оценки его сознания с применением традиционных шкал: West-Haven и шкалы комы Глазго.

Еще один важный аспект в оценке статуса пациента с циррозом печени в аспекте ПЭ - саркопения (истощение мышечной массы). Клетки скелетных мышц содержат большое количество глутамина. При саркопении за счет активного мышечного катаболизма увеличивается высвобождение глутамина, который под действием фермента глутаминазы расщепляется с образованием аммиака и приводит к гипераммониемии [4]. Согласно результатам проведенных к настоящему времени исследований, саркопения служит самостоятельным фактором жизненного прогноза этих пациентов и оказывает влияние на риск развития ПЭ.

\section{ПРИНЦИПЫ ЛЕЧЕНИЯ ПЕЧЕНОЧНОЙ ЭНЦЕФАЛОПАТИИ}

Доминирующая роль аммиака в патогенезе ПЭ при циррозе печени определяет основное направление лечения - уменьшение его концентрации, что достигается за счет снижения его продукции в кишечнике и увеличения элиминации гепатоцитами. Соответственно, в целях уменьшения продукции аммиака в кишечнике применяются следующие основные препараты: невсасывающийся антибиотик рифаксимин и осмотическое слабительное лактулоза (лактитол). Для стимуляции клиренса аммиака гепатоцитами (через образование мочевины и синтез глутамина) и/или мышечными клетками применяется L-орнитин-Lаспартат. Подробные схемы назначения этих препаратов указаны в клинических рекомендациях Российского общества по изучению печени по лечению пациентов с циррозом печени в разделе «лечение печеночной энцефалопа- тии» [2]. В отличие от европейских и американских рекомендаций в РФ любой из вышеперечисленных препаратов может быть использован в качестве терапии первой линии по усмотрению лечащего врача и анализа особенностей заболевания у конкретного пациента.

Лактулоза (лактитол) - слабительное средство, которое оказывает незначительное влияние на состав или функции кишечной микробиоты, ее эффект реализуется, вероятно, за счет ускорения кишечного транзита и подкисления кишечной среды. Вследствие этого продукция аммиака в кишечнике снижается, а его экскреция с калом увеличивается. Большое количество рандомизированных клинических испытаний, а также обсервационных исследований показали преимущество лактулозы в лечении ПЭ в сравнении с отсутствием терапии, хотя настоящих двойных слепых исследований нет, т. к. очень трудно «ослепить» слабительный эффект и типичный сладкий вкус данного препарата. Ограничением для клинического применения лактулозы служат ее побочные действия: диарея, тошнота и вздутие живота. Диарея может привести к нарушению электролитного баланса и даже к обострению ПЭ. Соответственно, прием лактулозы нужно начинать с 15-20 мл каждые 12 ч и постепенно индивидуально увеличить дозу для достижения цели: мягкий стул 2 раза в день.

Противомикробные агенты. Рифаксимин - невсасывающийся антибиотик, который вводится перорально и практически не приводит к развитию резистентности бактерий. Рифаксимин оказывает позитивное влияние на микробный состав кишки, что приводит к уменьшению продукции аммиака. Препарат имеет хороший профиль безопасности, может применяться длительно и не требует коррекции дозы у пациентов с печеночной и/или почечной недостаточностью. Лечение рифаксимином приводит к разрешению ПЭ и уменьшает летальность от данного осложнения. Комбинация рифаксимина с лактулозой эффективнее применения лактулозы в качестве монотерапии [4].

L-орнитин-L-аспартат (LOLA) изучался в большом количестве рандомизированных клинических исследований. В 2019 г. был опубликован обзор рандомизированных клинических исследований и метаанализов, в которых проанализирована эффективность LOLA у пациентов с циррозом печени в сравнении с плацебо, отсутствием каких-либо вмешательств, другими препаратами для лечения ПЭ (лактулозой, рифаксимином и т. д.). Внутривенное введение LOLA в дозе 20-40 мг в сутки в течение 7 дней в 3,22 раза, по сравнению с плацебо, улучшало психический статус пациентов с ЦП классов А и В по шкале Чайлд - Пью, уменьшало проявления ПЭ и снижало постпрандиальный уровень аммиака. Это свойство внутривенной формы препарата с успехом может использоваться в дифференциальном диагнозе ПЭ при циррозе печени с другими состояниями, которые влияют на психический статус пациентов, например синдром отмены алкоголя. Это особенно актуально в отделениях интенсивной терапии. В этой ситуации правильный диагноз строится по принципу “ех juvantibus”, поскольку гипераммониемия - ключевой фактор патогенеза ПЭ, а не синдрома отмены. 
Объединенный анализ всех исследований демонстрирует эффект как внутривенной, так и пероральной формы LOLA на течение как клинически выраженной, так и скрытой ПЭ, и на уровень аммиака в крови в сравнении с плацебо/отсутствием лечения. LOLA не уступает в эффективности лечения невсасывающемуся антибиотику рифаксимину и осмотическому слабительному лактулозе [11].

В программу лечения осложнений цирроза печени входит человеческий альбумин, его способность удалять токсические соединения из циркуляции актуальна в лечении ПЭ.

\section{СКРЫТАЯ ПЕЧЕНОЧНАЯ ЭНЦЕФАЛОПАТИЯ}

Отдельный вопрос, который постоянно обсуждается экспертами в области ведения пациентов с циррозом печени, - скрытая ПЭ. Этой теме большое внимание уделил консенсус ISHEN. Скрытая ПЭ или ПЭ легкой степени (к этой форме относится минимальная и ПЭ 1-й степени согласно критериям West-Haven) очень часто встречается в клинической практике. Как правило, пациенты со скрытой ПЭ находятся в амбулаторных условиях и занимаются привычными для себя делами. Однако скрытая ПЭ отрицательно влияет на качество жизни, социальные и семейные отношения, способность обучаться и эффективно работать, способность управлять автомобилем. Кроме того, она связана с более высокой вероятностью развития явной ПЭ. Поэтому согласно клиническим рекомендациям Американской и Европейской ассоциаций по изучению печени по ведению пациентов с ПЭ, целесообразно каждого пациента из группы риска развития данного состояния скринировать на предмет скрытой ПЭ. В нашей стране для диагностики скрытой ПЭ чаще всего используются такие психометрические тесты, как «тест связи чисел» и «тест линий». Они требуют наличия ручки и бумаги, а также определенного навыка. В последние годы скрининг ПЭ легкой степени стал проще, поскольку доступны такие инструменты, которые можно применять прямо у постели больного. Примером служит тест на клички животных, он универсален и быстро применим (не требует оборудования и занимает всего 60 сек). Данный тест может выполняться врачами, медсестрами и лицами, осуществляющими уход за пациентом, он имеет ограниченную спонтанную вариабельность, что делает его полезным для последующего динамического наблюдения/мониторинга. Суть этого теста заключается в том, что в течение 60 сек пациенту предлагается называть абсолютно произвольно клички домашних животных. Упрощенный тест на клички животных оценивается следующим образом: если в течение 60 сек пациент называет более 15 кличек животных, то энцефалопатии нет (присваивается значение 0), если за этот период времени он называет от 10 до 15 кличек животных, то есть легкая степень ПЭ (присваивается значение 1), если пациент способен назвать менее 10 кличек животных, то есть более значимая ПЭ и присваивается значение 2. Эта оценка коррелировала как с результатами психометрических тестов ( $p<0,0001)$, так и с данными электроэнцефалографии ( $p=0,007)$. Более того, данная трехуровневая оценка показала прогностическую ценность в отношении годичного риска развития явной ПЭ и смерти [12].

\section{ЗАКЛЮЧЕНИЕ И ПРАКТИЧЕСКИЕ РЕКОМЕНДАЦИИ}

Основные вопросы, которые дискутируются и по которым существуют рекомендации, согласно мнению экспертов и консенсусных документов в дополнение к уже разработанным гайдлайнам.

Диагностическая роль аммиака. В практической работе можно использовать следующие положения: у пациентов с подозрением на явную, клинически выраженную ПЭ нормальная концентрация аммиака может быть использована для исключения данного диагноза. В этом случае нужно искать другую причину энцефалопатии (алкогольная или другая интоксикация, первичное поражение центральной нервной системы и др.). Напротив, высокая концентрация аммиака в отсутствие признаков явной ПЭ не должна быть критерием диагноза и основанием для терапевтических мер.

Скрининг скрытой печеночной энцефалопатии, к которой относят минимальную и ПЭ 1-й степени представляется актуальным в связи с тем, что данное состояние нарушает качество жизни пациентов, создает опасность при вождении транспорта и служит фоном для развития клинически явной ПЭ. Самый простой и доступный тест, который может выполнить как врач, так и человек, ухаживающий за пациентом, - это тест на клички животных. Он не подвержен вариабельности, не требует никаких дополнительных инструментов и оказывает помощь в оценке эффективности проводимой терапии.

В беседу с пациентами, страдающими минимальной ПЭ, рационально добавлять вопросы: «Вы водите машину?», «Были ли у вас аварии или опасные ситуации»? Особое внимание следует уделять оценке когнитивной функции пациентов с циррозом, которые активно водят машину и/или недавно (<3 мес. назад) перенесли эпизод клинически выраженной ПЭ.

Пациентам с минимальной ПЭ необходимо проводить лечение по тем же принципам, что и пациентам с явной ПЭ, исходя из аналогичного патогенеза и риска ее прогрессирования

Основные препараты для лечения ПЭ - лактулоза, рифаксимин и L-орнитин-L-аспартат.

Диагностика явной ПЭ и методы ее терапии четко разработаны. Согласно российским рекомендациям, в зависимости от особенностей течения заболевания у конкретного пациента в качестве препаратов первой линии могут быть использованы лактулоза, рифаксимин, L-орнитин-L-аспартат.

Внутривенная форма L-орнитина-L-аспартата оказывает большую помощь в дифференциальном диагнозе явной, тяжелой ПЭ с другими формами энцефалопатии, поскольку эффект на введение данного препарата в виде улучшения сознания при ПЭ развивается очень быстро.

Поступила / Received 30.08.2021 Поступила после рецензирования / Revised 15.09.2021 Принята в печать / Accepted 16.09.2021 
1. Vilstrup H., Amodio P., Bajaj J., Cordoba J., Ferenci P., Mullen K.D. et al. Hepatic encephalopathy in chronic liver disease: 2014 Practice Guideline by the American Association for the Study of Liver Diseases and the European Association for the Study of the Liver. Hepatology. 2014;60(2):715-735. https://doi.org/ 10.1002/hep.27210.

2. Ивашкин В.Т., Маевская М.В., Павлов Ч.С., Федосьина Е.А., Бессонова Е.Н., Пирогова И.Ю., Гарбузенко Д.В. Клинические рекомендации Российского общества по изучению печени и Российской гастроэнтерологической ассоциации по лечению осложнений цирроза печени. Российский жур нал гастроэнтерологии, гепатологии, колопроктологии. 2016;26(4):71102. Режим доступа: https://www.gastro-j.ru/jour/article/view/73. Ivashkin V.T., Mayevskaya M.V., Pavlov Ch.S., Fedosyina Y.A., Bessonova Y.N., Pirogova I.Yu., Garbuzenko D.V. Treatment of liver cirrhosis complications: Clinical guidelines of the Russian Scientific Liver Society and Russian gastroenterological association. Rossiyskiy zhurnal gastroehnterologii, gepatologii, koloproktologii = Russian Journal of Gastroenterology, Hepatology, Coloproctology. 2016;26(4):71-102. (In Russ.) Available at: https://www.gastro-j. ru/jour/article/view/73.

3. Bajaj J.S., Lauridsen M., Tapper E.B., Duarte-Rojo A., Rahimi R.S., Tandon P. et al. Important Unresolved Questions in the Management of Hepatic Encephalopathy: An ISHEN Consensus. Am J Gastroenterol. 2020;115(7):989-1002. https://doi.org/10.14309/ajg.0000000000000603.

4. Rose C.F., Amodio P., Bajaj J.S., Dhiman R.K., Montagnese S., TaylorRobinson S.D. et al. Hepatic encephalopathy: Novel insights into classification, pathophysiology and therapy. J Hepatol. 2020;73(6):1526-1547. https://doi.org/10.1016/j.jhep.2020.07.013

5. Garcia-Pagan J.C., Francoz C., Montagnese S., Senzolo M., Mookerjee R.P. Management of the major complications of cirrhosis: Beyond guidelines. J Hepatol. 2021;75(1 Suppl.):S135-S146. https://doi.org/10.1016/j. jhep.2021.01.027.
6. Сиволап Ю.П. Профилактика и лечение печеночной энцефалопатии Журнал неврологии и психиатрии им. С.С. Корсакова. 2017;117(10):144-147. https://doi.org/10.17116/jnevro2017117101144-147.

Sivolap lu.P. Prevention and treatment of hepatic encephalopathy. Zhurnal Nevrologii i Psikhiatrii imeni S.S. Korsakova = S.S. Korsakov Journal of Neurology and Psychiatry. 2017;117(10):144-147. (In Russ.) https://doi.org/10.17116/ jnevro2017117101144-147.

7. Bhatia V., Singh R., Acharya S.K. Predictive value of arterial ammonia for complications and outcome in acute liver failure. Gut. 2006;55(1):98-104. https://doi.org/10.1136/gut.2004.061754.

8. Shalimar, Sheikh M.F., Mookerjee R.P., Agarwal B., Acharya S.K., Jalan R. Prognostic Role of Ammonia in Patients With Cirrhosis. Hepatology. 2019;70(3):982-994. https://doi.org/10.1002/hep.30534

9. Gundling F., Zelihic E., Seidl H., Haller B., Umgelter A., Schepp W., Dodt C. How to diagnose hepatic encephalopathy in the emergency department. Ann Hepatol. 2013;12(1):108-114. Available at: https://pubmed.ncbi.nlm. nih.gov/23293201/.

10. Bajaj J.S., Bloom P.P., Chung R.T., Hassanein T.I., Padilla-Martinez M., Kayali Z. et al. Variability and Lability of Ammonia Levels in Healthy Volunteers and Patients With Cirrhosis: Implications for Trial Design and Clinical Practice. Am J Gastroenterol. 2020;115(5):783-785. https://doi.org/10.14309/ ajg.0000000000000384.

11. Goh E.T., Stokes C.S., Sidhu S.S., Vilstrup H., Gluud L.L., Morgan M.Y. L-ornithine L-aspartate for prevention and treatment of hepatic encephalopathy in people with cirrhosis. Cochrane Database Syst Rev. 2018;5(5):CD012410. https://doi.org/10.1002/14651858.cd012410.pub2.

12. Campagna F., Montagnese S., Ridola L., Senzolo M., Schiff S., De Rui M. et al. The animal naming test: An easy tool for the assessment of hepatic encephalopathy. Hepatology. 2017;66(1):198-208. https://doi.org/10.1002/ hep. 29146

\section{Информация об авторе:}

Маевская Марина Викторовна, д.М.н., профессор кафедры пропедевтики внутренних болезней, гастроэнтерологии и гепатологии Института клинической медицины имени Н.В. Склифосовского, Первый Московский государственный медицинский университет имени И.М. Сеченова (Сеченовский Университет); 119991, Россия, Москва, ул. Погодинская, д. 1, корп. 1; mvmaevskaya@me.com.

\section{Information about the author:}

Marina V. Maevskaya, Dr. Sci. (Med.), Professor of Chair for Internal Diseases Propedeutics, Gastroenterology and Hepatology, Sklifosovsky Clinical Medicine Institute, Sechenov First Moscow State Medical University (Sechenov University); 1, Bldg. 1, Pogodinskaya St., Moscow, 119991, Russia; mvmaevskaya@me.com 